\title{
STABILITY OF INTERSECTING AIRCRAFT FLOWS UNDER DECENTRALIZED CONFLICT AVOIDANCE RULES *
}

\author{
Zhi-Hong Mao ${ }^{\dagger}$ and Eric Feron $\ddagger$ \\ Massachusetts Institute of Technology, Cambridge, MA 02139 \\ Karl Bilimoria \& \\ NASA Ames Research Center, Moffett Field, CA 94035
}

\begin{abstract}
$\underline{\text { Abstract }}$
This paper considers the problem of intersecting aircraft flows under decentralized conflict avoidance rules. Using an Eulerian standpoint (aircraft flow through a fixed control volume), new air traffic control models and scenarios are defined that enable the study of long-term airspace stability problems. Considering a class of two intersecting aircraft flows, it is shown that airspace stability, defined both in terms of safety and performance is preserved under specific decentralized conflict resolution algorithms. A performance bound is derived and examples show that this bound is tight. A comparison is made with centralized approaches to conflict resolution.
\end{abstract}

\section{$\underline{\text { Introduction }}$}

The air transportation system is currently the object of intensive research, following the sustained growth of past and forecasted air traffic. The current enroute air traffic control system consists of the following elements:

- A geographical network whose nodes are navigation beacons (VHF Omnidirectional Range (VOR) and Distance Measuring Equipment systems (DME)), and whose links are air routes. The aircraft are allowed to fly only along these routes (with some exceptions). Flying on segments connecting two navigation beacons makes the problem of aircraft navigation and automated guidance particularly easy.

\footnotetext{
* Copyright (c) 2000 The American Institute of Aeronautics and Astronautics, Inc. All rights reserved.

${ }^{\dagger}$ Research assistant, Department of Aeronautics and Astronautics

$\ddagger$ Senior Member, AIAA. Associate Professor, Department of Aeronautics and Astronautics. Author to whom all correspondence should be sent. feron@mit.edu

$\S$ Associate Fellow, AIAA. kbilimoria@mail.arc.nasa.gov
}

- Approximately 1500 enroute air traffic controllers who regulate the aircraft flow across this network and make sure no hazardous situation develops, whereby two aircraft might get too close to each other (aircraft conflicts). The network structure of the aircraft routing system allows the controllers to get a priori information on aircraft conflict geometries and their location during nominal operations: Conflicts are usually located at the nodes of the network. Knowing potential conflict locations a priori enables the decomposition of the airspace into sectors, managed by individual air traffic controllers, and whose boundaries are located away from the network nodes and therefore away from the most common conflict locations.

Many decades of working experience have demonstrated that this network-based architecture is safe. However, it suffers from strong perceived drawbacks, such as systematic indirect routing between origin and destination, and in general a perceived lack of navigation freedom for the pilots. The advent of a relatively new generation of Global Navigation Satellite Systems (GNSS), in particular GPS, has removed in principle the limitations of the ground-based navigation infrastructure. In particular, it is now very easy to obtain precise aircraft position anywhere over the United States and not only on a pre-determined set of routes (although this idea, also named Area Navigation, has been demonstrated to be feasible for many years [3], using the conventional navigation infrastructure, at the expense of improved on-board computational equipment). As a consequence, concepts of operations such as "Free Flight" [20] have been proposed by airlines and by the Federal Aviation Administration (FAA) to remove the routing constraints imposed by the conventional, fixed-route system. Under Free Flight, each aircraft would be able to optimize its trajectory according to several factors such 
as perceived safety, weather, direct operating costs and coordination with other flights [19]. Some steps towards Free Flight include the National Route Program, whereby qualified aircraft are allowed to fly their preferred route after approval by the air traffic services. However, in order to be implemented on a full scale, the safety of such concepts needs to be proven. In particular, the set of standards over which operational concepts are evaluated has evolved from empirical evaluation decades ago to a sophisticated and very difficult certification process, which makes proving the safety of any new concept of operations a very challenging task. While many years of reliable operation provide evidence of safety for the current air traffic control system, the safety of any new system cannot rely on experience only, as it is very lengthy and expensive to build up. Rather, future air traffic management concepts will draw from appropriate mathematical modeling and engineering analysis techniques. Thus, Free Flight offers a wide array of new challenges and opportunities to the research community.

This paper considers the problem of air traffic "stability" under decentralized conflict detection and resolution rules. However, one of the major issues arising when considering this problem is the proper definition of "stability" of air traffic flows. In traditional control system terms, the notion of "stability" usually relates to the long term behavior of dynamical systems, which is expected to remain within some acceptable bounds and often to converge towards a specific desired state. For example, individual aircraft stability concepts are tied to the requirement that both aircraft attitude and position stay close enough to some reference attitude and position.

Considering problems of air traffic management, the requirement for stability becomes more complex: While aircraft are expected to follow a reference trajectory (as loosely defined as it may be), aircraft are also required to stay away from each other to prevent near misses or even airborne collisions. In this context it becomes quite important for the researcher to define appropriate notions of stability. This in turn entails the requirement of appropriately defining the system being worked upon. Much of the current research focuses on problems involving a finite, usually small number of aircraft. Such a Lagrangian standpoint (in which few aircraft are analyzed) is useful when designing efficient conflict detection and resolution systems. However, this standpoint is not convenient to use for flow stability analyses, since interactions occurring within a finite set of aircraft can only have a finite duration.

We propose in this paper an "Eulerian" standpoint, whereby an arbitrarily large number of aircraft flows through an otherwise well-defined airspace volume. The motivation behind this standpoint is that, even under Free Flight, many aircraft flow interactions are expected to occur within relatively welldefined parts of the airspace, corresponding to the intersection between one or more optimal routes linking city pairs, for example. This viewpoint is also very much compatible with an air traffic controller's current view of the air transportation system, with the volume of airspace being a sector. Note this standpoint also appears in [5].

This paper is organized as follows: First, the aircraft flow models are introduced. An appropriate notion of aircraft flow stability is defined and the decentralized strategy followed by each aircraft is detailed. Second, a proof of interacting aircraft flow stability is provided for the case of two intersecting aircraft flows where aircraft use a simple, decentralized conflict resolution rule. Third, a discussion of the results is presented, along with simulations. A comparison is drawn between centralized and decentralized conflict resolution.

\section{$\underline{\text { Air Traffic Models and Problem Formulation }}$}

$\underline{\text { General Considerations }}$

The definition of appropriate models appears to be a significant challenge when considering problems in air transportation [9]. Considering the conflict detection and resolution problem, most authors (including those of this paper) have traditionally concentrated on scenarios involving a finite number of aircraft. However, there appears to be a widespread concern about the "domino effect", whereby one conflict resolution maneuver creates new conflicts which in turn need to be solved etc. In this paper, we will therefore concentrate on a possibly infinite number of aircraft flowing through a finite portion of the airspace.

The system under study consists of a given volume of airspace, and a set of aircraft flowing in and out of it, as shown in Fig. 1. The dynamics of the system are determined by the "boundary conditions" that indicate the location, speed and rate at which aircraft appear in the volume of airspace, and by their individual behavior while they fly within the airspace. Clearly, some boundary conditions are unacceptable, e.g., the case when two aircraft appear into the control region very close to each other and on a head-on collision course. Since relatively little is known about 


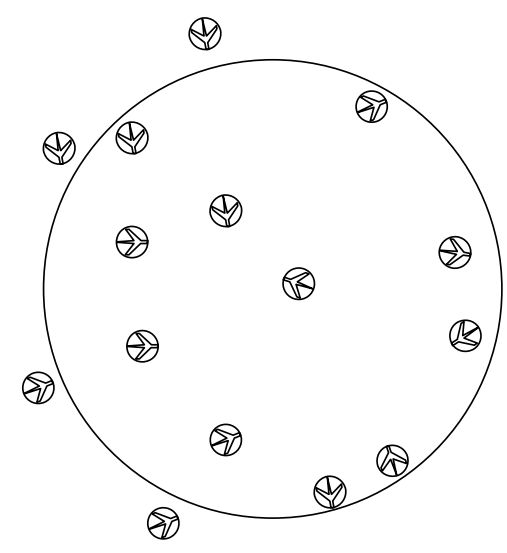

Figure 1: Aircraft flowing in and out of "control volume".

interacting aircraft flows, this paper will consider aircraft flows with low complexity.

The aircraft are assumed to be intelligent, that is, their pilots actively attempt to maneuver and avoid conflicts at the smallest possible cost.

\section{$\underline{\text { Aircraft Maneuver Models }}$}

Although designing and analyzing systems for aircraft conflict detection and resolution needs to account for the three dimensions, this paper will only investigate air traffic evolving in two dimensions (planar conflict resolution): The trajectories of all aircraft are assumed to evolve in the horizontal plane. While vertical maneuvers appear to be most efficient for tactical conflict resolution (such as in the case of TCAS (Traffic Alert and Collision Avoidance System)), horizontal maneuvers might be more appropriate for the "strategic" conflict resolution context considered in this paper, because they induce less passenger discomfort and they do not require flight level changes and thus may not perturb the vertically stratified traffic structure as it exists today in the enroute airspace.

This paper will be concerned with very simple aircraft behaviors. In particular aircraft fly only along straight, level and constant speed trajectories. All aircraft have the same absolute speed. Moreover, we will assume that only one conflict area exists, and that aircraft may perform only one conflict avoidance maneuver [2]. In this paper we will be concerned with maneuvers involving no speed change.

Two models for conflict avoidance will be considered in this paper; Fig. 2 illustrates these conflict resolution models.

- Velocity change model: In this model (left picture in Fig. 2), single heading changes are used

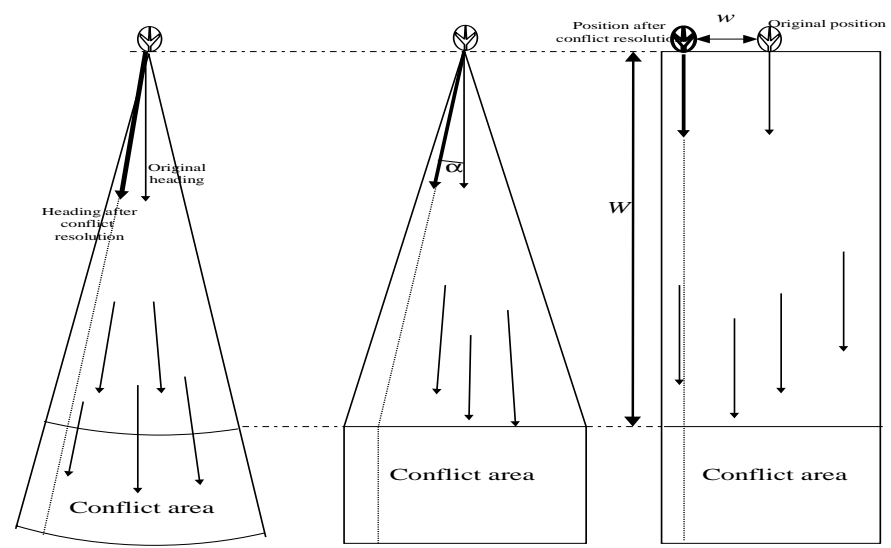

Figure 2: Heading change model v.s. position change model. Left: The aircraft maneuver is an immediate heading change. Middle: The aircraft maneuver is a heading change followed by a second heading change. Right: The aircraft maneuver is an immediate position change.

to modify aircraft trajectories. Following the approach of Andrews [2], these changes are assumed to occur instantaneously when the aircraft makes a decision. This model will be used for simulation purposes.

- Position change model: This model (right picture in Fig. 2), used for simulation and analysis purposes, consists of modeling aircraft trajectory changes via a single and instantaneous lateral position change, the speed vector remaining the same before and after the position change. This model appears to be less realistic; However, it is simpler to use for analysis purposes. In addition, the position change model can be treated as a close approximate of the two-stage maneuver model shown in the middle of Fig. 2. Given the distance $W$, the lateral displacement $w$ in the position change model is equivalent to a heading change of amplitude $\alpha=\tan (w / W)^{-1}(\approx$ $w / W$ if $W$ is much greater than $w$, which is usually the case for strategic conflict resolution.) The longitudinal displacement difference between these two maneuver models is on the order of $w^{2} / W$, which will be assumed to be small. In the following, $W$ will represent a distance to a conflict area shown in Fig. 2. The advantage of this maneuver model is its simplicity of use for analysis purposes.

\section{$\underline{\text { Aircraft Flow Arrival Geometry }}$}

The basic aircraft flow model chosen in this paper is that shown in Fig. 3. and it was originally intro- 


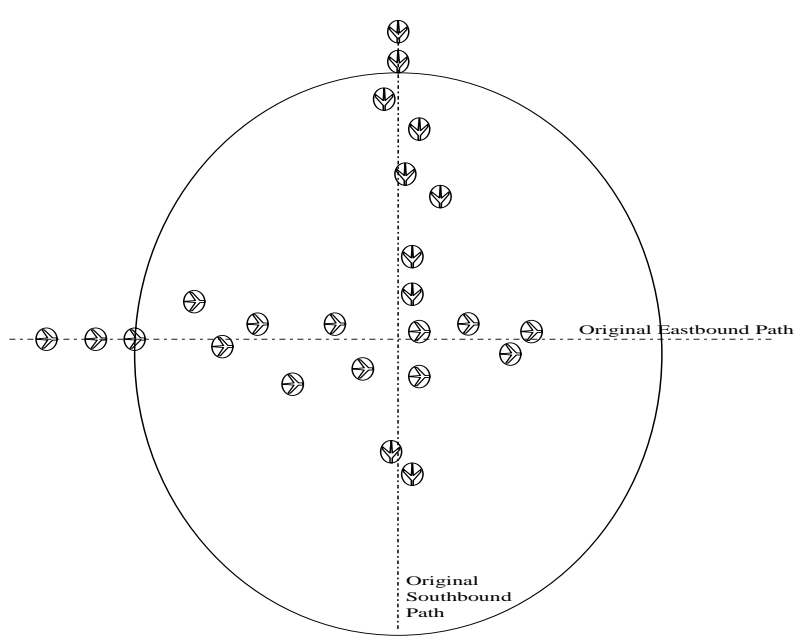

Figure 3: Two crossing aircraft flows.

duced by Niedringhaus [15]. Two aircraft streams, oriented at a given angle $\theta(\theta=90$ degrees in Fig. 3) relative to each other, feed aircraft into a circular conflict area. The streams are organized in such a way that all aircraft within each stream are originally headed in the same direction. For simplicity of exposition only, it will also be assumed all aircraft are originally flying along the same track prior to entering the control volume. The spacing between each aircraft in each flow is arbitrary but no less than a given minimum safe distance $d$. Let $A_{1}, A_{2}, \ldots, A_{i}, \ldots$ be the set of aircraft entering the control volume, where aircraft are indexed according to the order they entered the control volume. When two aircraft enter the control volume at the same time, the southbound aircraft is indexed first.

\section{Conflict Resolution Rules}

Several centralized and decentralized conflict resolution rules are available (see for example $[7,8,16$, 10, 11, 12, 13, 14, 18, 22, 23]).

A conflict is declared when the projected straight path of any aircraft pair leads them to a miss distance less than a preset threshold $d$. The decentralized conflict resolution scheme chosen in this paper follows a sequential approach, whereby aircraft solve potential conflicts one at a time. To simplify matters, it is assumed that the order in which aircraft perform their resolution maneuver is the same as the order they enter the circular conflict area, although this assumption could be relaxed. An aircraft solving a conflict considers all other aircraft that maneuvered before it as moving obstacles, but does not account for the aircraft which have not maneuvered yet. Thus each aircraft has knowledge of all aircraft that have already performed a maneuver (or decided that no maneuver was necessary). A reliable implementation of such sequential approaches have been described in [1].

\section{$\underline{\text { Conflict Resolution Maneuver }}$}

Given those aircraft $A_{1}, \ldots, A_{i-1}$ which have already performed a resolution maneuver (and must therefore be considered as obstacles), the resolution maneuver for the next aircraft $i$ scheduled for conflict resolution will be such that (i) no conflict exists between $A_{i}$ and $A_{i}, \ldots, A_{i-1}$ after the resolution and (ii) the amplitude of the conflict avoidance maneuver is as small as possible. For the heading change model, the resolution maneuver will be to minimize the amplitude of the deviation from the nominal heading. Similarly, for the lateral position change model, the resolution maneuver will attempt to minimize the lateral position change necessary for conflict resolution. In the computer simulations presented thereafter, both conflict resolution maneuvers (heading change and position change) are generated by a simple line search away from the nominal heading and position, using a predefined step size.

\section{Notions of Stability}

In this paper, we will consider the system to be stable if:

- All conflicts get resolved without creating the "domino effect", whereby a conflict resolution maneuver creates a cascading series of new conflicts that propagate through the airspace.

- The deviation of the aircraft trajectory from nominal, due to the requirement for conflict resolution, is bounded. In other words, the heading change is bounded or the lateral position change is bounded, depending upon which aircraft maneuver model is chosen.

This definition summarizes the two most important requirements in air traffic control: Guaranteed safety and efficiency of traffic handling.

\section{Closed-loop $\underline{\text { System Stability }}$}

This section presents the main result of this paper: The system shown in Fig. 3, under the conflict resolution rule described above, is stable. More precisely, we ask the following question: Assuming the system has been running correctly in the past, will it keep running correctly in the future? Indeed, it is possible 


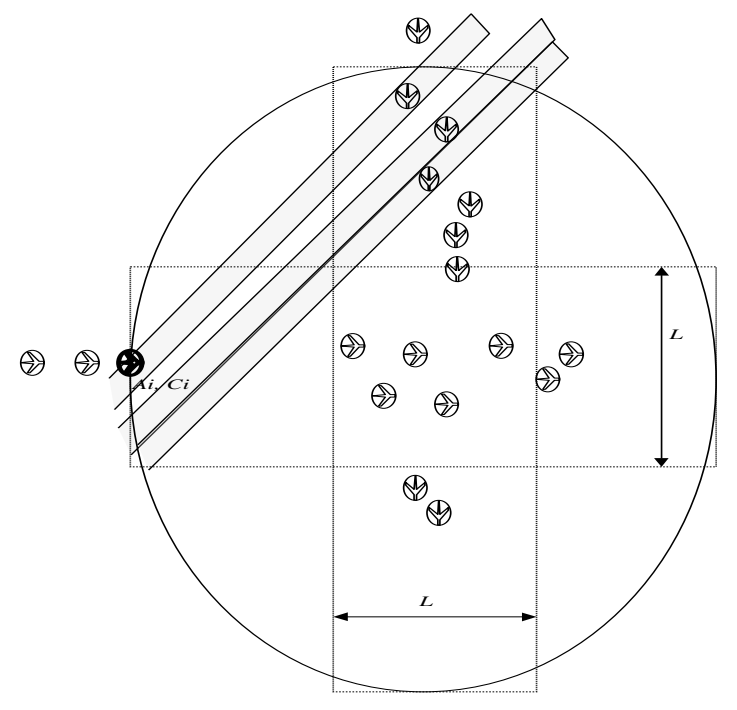

Figure 4: Aircraft flow configuration for stability analysis. The shaded areas are locations where a conflict will occur.

to construct "initial conditions" for the system such that conflicts are unavoidable. We now show that an incoming aircraft (shown in bold in Fig. 4) can always find a conflict resolution maneuver and proceed with a conflict-free trajectory. In this section, we will assume that aircraft resolve conflicts via position changes.

\section{$\underline{\text { Conflict Geometry }}$}

For simplicity, we will assume that the two crossing aircraft flows are oriented 90 degrees with respect to each other, with one southbound and one eastbound aircraft flow, as shown in Fig. 4. Also, we will assume that all aircraft initially follow each other along two intersecting lines (one eastbound, one southbound), and that after maneuvering, all aircraft remain within a "maneuver corridor" of total width $L$ centered along the nominal paths. These assumptions may be relaxed to an arbitrary encounter angle.

Without loss of generality, one may assume that the next aircraft $A_{i}$ to perform a resolution maneuver is eastbound, as represented in bold in Fig. 4. By definition of the aircraft flow and allowable maneuvers, this aircraft never conflicts with neighboring eastbound aircraft. In addition, each southbound aircraft within the control volume has already performed a resolution maneuver and must be considered as a moving obstacle. Each southbound aircraft projects a linear, slab-shaped "shadow" of width $d$, centered around the aircraft and inclined 45 degrees (denoted in light grey in Fig. 4). For general encounter angles and aircraft speeds, this shadow is oriented along the relative velocity vectors. Consider a circle $C_{i}$ of radius $d / 2$ centered around $A_{i}$ (note that the size of

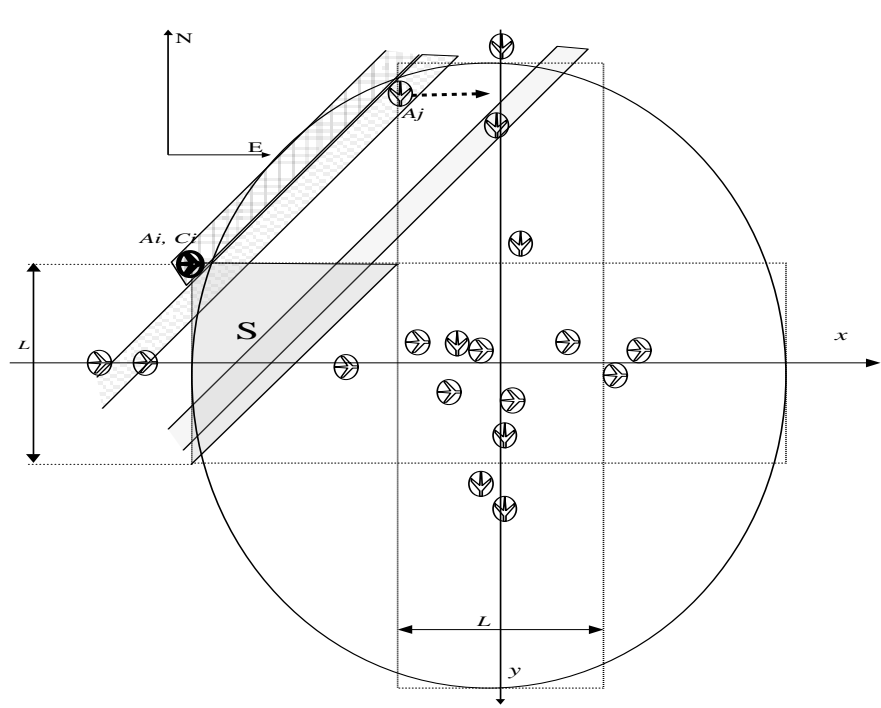

Figure 5: Existence of conflict resolution maneuver is enable by intelligent behavior.

the aircraft drawn in all figures is considerably exaggerated). For the eastbound aircraft $A_{i}$ to avoid any conflict, it must maneuver so that the circle $C_{i}$ does not intersect any of the shaded areas. Failure to do so means that a conflict will occur.

\section{Existence of Conflict Resolution Maneuver}

It is now shown that an aircraft entering the control volume (e.g., the eastbound aircraft $A_{i}$ indicated in bold in Fig. 5) can always execute a lateral displacement maneuver that results in a conflict-free trajectory, if the width of the maneuver corridor, $L$, is sufficiently large. We begin with the hypothesis that such a maneuver does not exist, and then make the following argument:

The "shadows" of the eastbound aircraft which are ahead of $A_{i}$ should not cover the circle $C_{i}$ (centered around aircraft $A_{i}$ ), wherever $A_{i}$ is located within the maneuver corridor. Otherwise, aircraft $A_{i}$ could hide behind one of such "shadows", and therefore, succeed in finding a lateral displacement (less than $L$ ) that results in a conflict-free trajectory. In other words, there should not exist any aircraft other than $A_{i}$ in the area of the shaded right triangle $S$ as seen in Fig. 5 .

At the same time, all southbound aircraft currently inside the control volume have already performed minimum lateral displacement conflict resolution maneuvers, and are flying along straight, conflictfree southbound paths. By hypothesis, their "shadows" intersect the circle $C_{i}$, wherever its location is, since no conflict resolution is possible. In particular, at least one "shadow" intersects the circle $C_{i}$ when $A_{i}$ deviates fully to the left, as shown in Fig. 5. For 

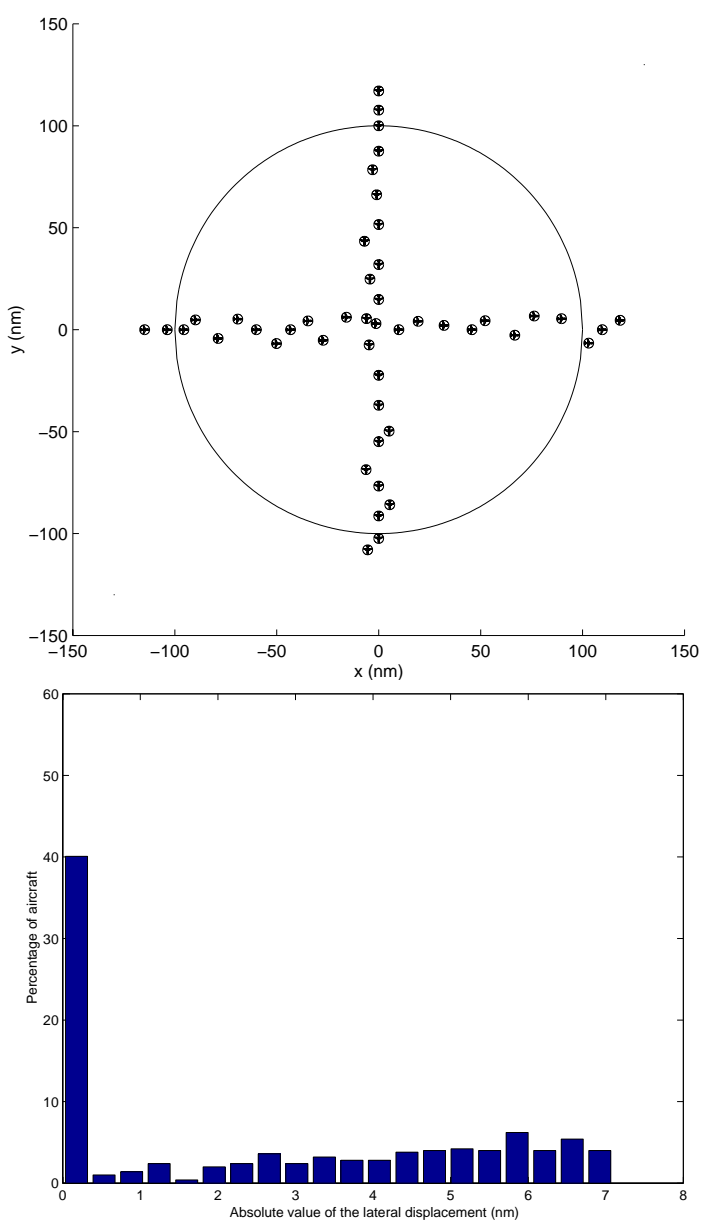

Figure 6: Test case for random arrival geometry using the lateral position change model. The separation distance is subject to a uniform distribution on the interval $[5,15]$ $\mathrm{nm}$. The number of the tested aircraft is 500. Upper: A snapshot taken during the conflict resolution process. Lower: Aircraft deviation distribution.

this to happen, the corresponding southbound aircraft (denoted by $A_{j}$ ) must have deviated to the right (from its nominal path) by a distance larger than $L / 2-\sqrt{2} d$.

Assume

$$
L>2 \sqrt{2} d .
$$

From the above argument about the eastbound aircraft, we see that the "shadows" of the eastbound aircraft should not intersect the circle $C_{j}$ (centered around $A_{j}$ ) if $A_{j}$ were shifted a lateral displacement of $L / 2-\sqrt{2} d$ closer to the central axis of the maneuver corridor. Thus, we get a conflict-free resolution maneuver for $A_{j}$ with smaller amplitude. This causes contradiction with the statement that the southbound aircraft have already performed minimum lateral displacement maneuvers.
Therefore, there must exist a conflict avoidance maneuver for $A_{i}$, and the closed-loop system is therefore stable.

Note that condition (1) provides an upper bound on the maximum conflict avoidance maneuver amplitude for both southbound and eastbound aircraft: The maximum lateral deviation experienced by the aircraft is bounded above by $\sqrt{2} d$. If $d=5 \mathrm{~nm}$ (nautical miles), the lateral deviations will not be larger than $7.1 \mathrm{~nm}$. Considering a scenario where conflicts are predicted and solved 20 minutes ahead of time, and aircraft flying at 500 knots, the corresponding distance to conflict $D$ is approximately $160 \mathrm{~nm}$. Thus an equivalent maximum heading change amplitude would be approximately 2.5 degrees.

Now let us look at a very simple situation involving only two aircraft: An eastbound aircraft and a southbound aircraft arrive in the conflict area at the same time. Without loss of generality, we assume that the southbound aircraft makes decision and maneuver first. Since in our conflict resolution rules an aircraft does not account for the aircraft which have not maneuvered yet, the southbound aircraft need not take any action to avoid the conflict. Hence the conflict resolution is left to the eastbound aircraft only. It is easy to test that the minimum lateral displacement for the eastbound aircraft to avoid the conflict is exactly $\sqrt{2} d$. This example shows that the bound we obtained is tight. In general, it was observed that for the class of problems considered in this paper, up to six aircraft can be involved in the same conflict.

\section{Arbitrary Encounter Angle}

Let $\theta$ be the encounter angle between aircraft flows: $\theta=0$ deg corresponds to the case when the two flows are parallel and $\theta=180 \mathrm{deg}$ corresponds to the two flows going opposite directions.

A slight generalization of the previous reasoning allows us to prove that for general flow encounter angles, the lateral deviation of each aircraft is bounded above by

$$
\frac{d}{\sin (\theta / 2)}
$$

\section{$\underline{\text { Simulations }}$}

This section presents simulations of traffic under the conditions described above. The goal of the simulations is to estimate lower bounds on the maximum deviations actually experienced by the intersecting aircraft flows, as well as to generate some insight about the structure of the traffic flow after resolution. 


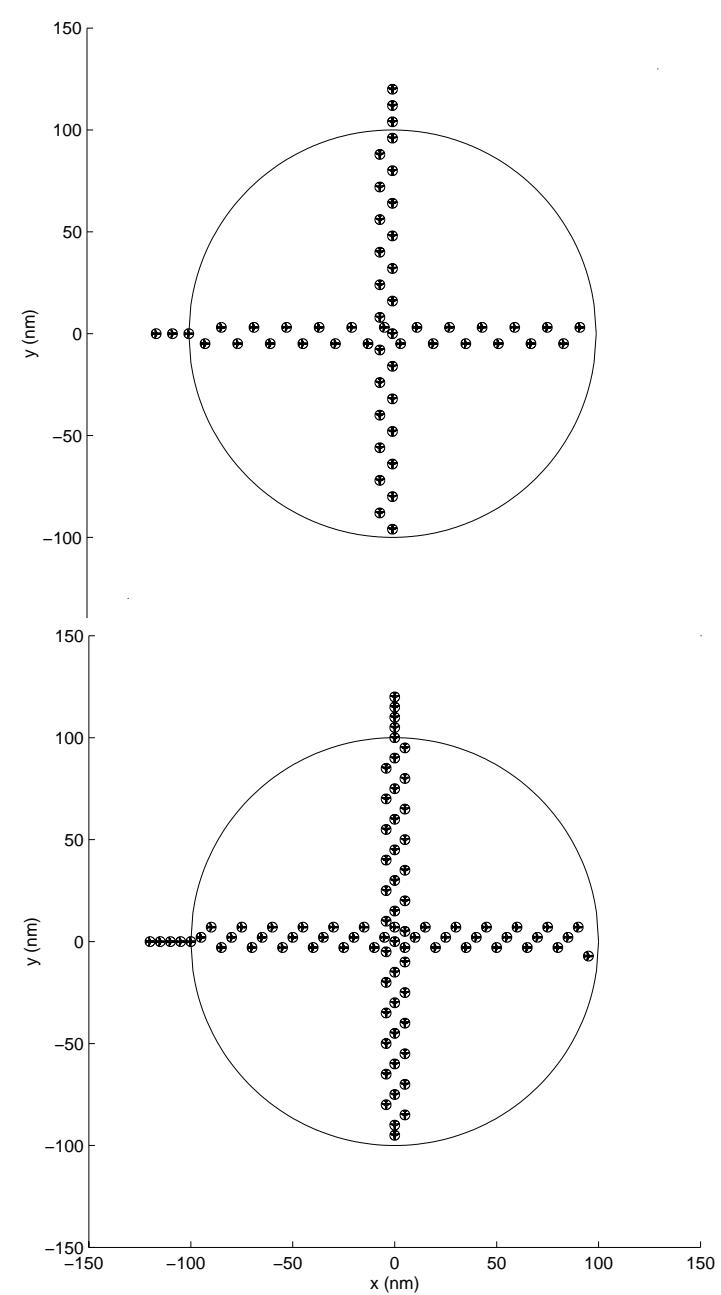

Figure 7: Test case for uniform arrival geometry using the position change model. Upper: Initial separation distance is $8 \mathrm{~nm}$. Lower: Initial separation distance is $5 \mathrm{~nm}$.

In this section, we will assume in the first two subsections that the aircraft resolve conflicts via lateral position changes. Some simulations and discussion for heading change maneuvers are given in the third subsection.

\section{$\underline{\text { Random Arrival Geometry }}$}

We first examine the lateral displacement of aircraft in the two intersecting streams for random arrival patterns. The aircraft in either stream are initially separated by a distance chosen from a uniform distribution over the interval $[5,15] \mathrm{nm}$. The considered airspace volume (conflict area) is circular with radius $100 \mathrm{~nm}$. A total of 500 aircraft flowing through this airspace have been simulated. Fig. 6 gives a snapshot of the traffic flow taken during the conflict resolution process. Also shown in Fig. 6 is a histogram of the lateral deviations experienced by the 500 tested aircraft. The largest lateral displacement found in this simulation is $7.1 \mathrm{~nm}$, which exactly is our estimated upper bound. The average of the (absolute) lateral displacements of the tested aircraft is $2.68 \mathrm{~nm}$, and there are $42.6 \%$ of the 500 tested aircraft deviating from the nominal path with a lateral displacement larger than half of the upper bound (approximately $3.5 \mathrm{~nm})$.

\section{$\underline{\text { Uniform Arrival Geometry }}$}

The following simulations consider the conflict resolution for two streams of aircraft with fixed initial separation distance. Although this uniform aircraft arrival geometry is unrealistic, it may help us get some intuition (in addition to the stability analysis) about how the proposed avoidance rules can successfully handle the conflict resolution for two streams of aircraft.

Fig. 7 gives two examples. In the first example, the initial separation distance between every pair of neighboring aircraft is $S=8 \mathrm{~nm}$. Denote $d_{s, 0}$ as the initial distance between the first aircraft in the southbound stream and the center of the conflict area, and, correspondingly, denote $d_{e, 0}$ for the first aircraft in the eastbound stream. In this example, $d_{s, 0}$ and $d_{e, 0}$ are chosen such that $d_{s, 0}-d_{e, 0}=S / 2$. Thus aircraft enter into the control volume one at a time. Fig. 7 (upper picture) shows the structure of the traffic flow after conflict resolution. The largest lateral displacement experienced by the aircraft is $6.2 \mathrm{~nm}$, within our estimated upper bound.

In the next example, we chose the initial separation distance of aircraft to be $S=d=5 \mathrm{~nm}$, which implies that in this case the aircraft are "packed" in the most compact way before they flow into the conflict area. We determined the behavior of the flows as $d_{s, 0}-d_{e, 0}$ decreases to 0 , that is, eastbound and southbound aircraft enter the control volume nearly at the same time. Fig. 7 (lower picture) presents a snapshot of the flows for this case during the conflict resolution process. The largest lateral displacement of the aircraft is $7.1 \mathrm{~nm}$.

From the above two examples we also observe that uniform aircraft arrival flows generate periodic, conflictfree aircraft flow patterns under the simple conflict avoidance rule. Furthermore, the conflict avoidance rule groups the aircraft in "platoons", and each platoon is formed in such a manner that the aircraft in a platoon have the same "shadow" as that of other aircraft in the same platoon. Intuitively, this kind of platooning is very efficient for conflict resolution in- 

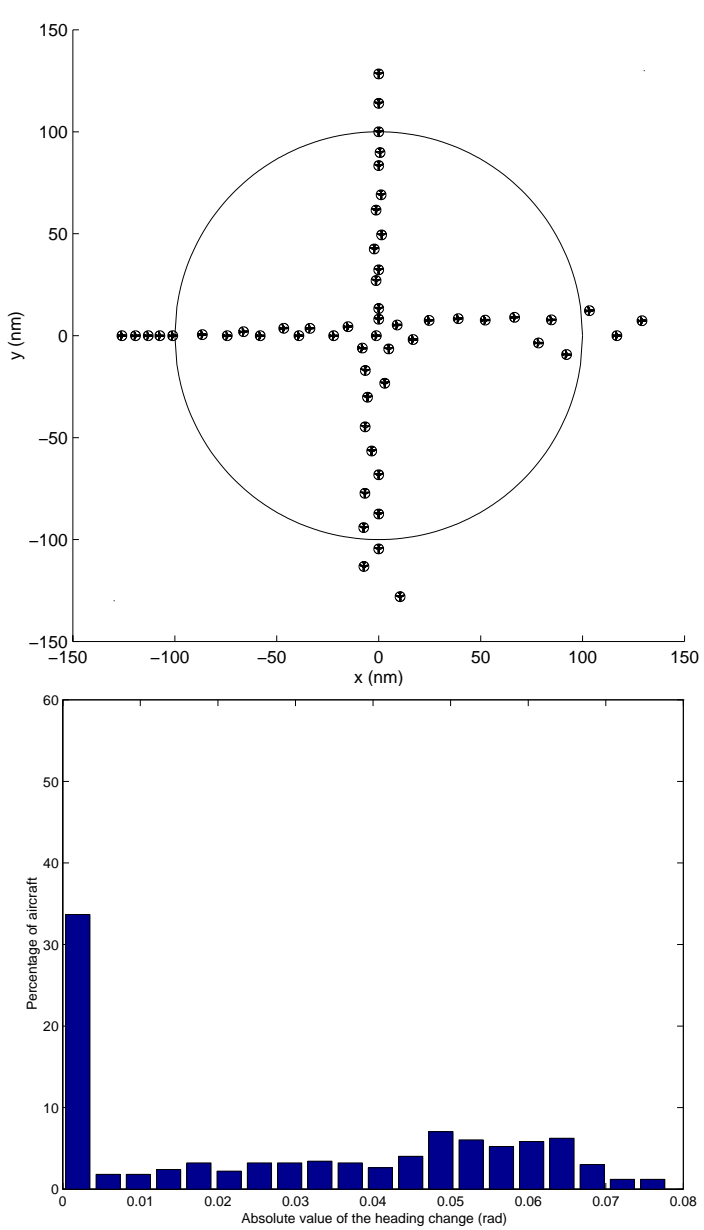

Figure 8: Test case for random arrival geometry using the heading change model. The separation distance is subject to a uniform distribution on the interval $[5,15] \mathrm{nm}$, the number of the tested aircraft is 500, and the radius of the conflict area is $100 \mathrm{~nm}$. Upper: A snapshot taken during the conflict resolution process. Lower: Aircraft deviation distribution.

volving two aircraft flows. The platooning results in a "shearing" motion when two platoons (from the two aircraft streams) meet at the center of the conflict area. Interesting enough, platooning has been proposed as a viable, although heuristic option in many intelligent, hierarchical transportation systems $[24,6]$.

\section{Stability Results for Heading Change Maneuver Model}

As mentioned in the previous sections, the lateral position change model may be considered as an approximation to the heading change model. The approximation accuracy can be examined by comparing the reachable position sets (areas) of both models for a given time horizon interval $[T, T+\Delta T]$. Besides

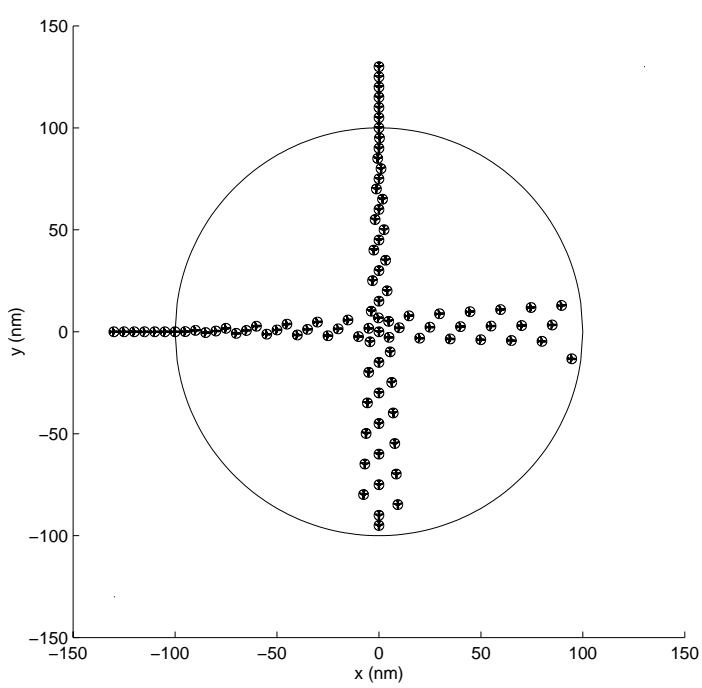

Figure 9: Test case for uniform arrival geometry using the heading change model. The initial separation distance is $5 \mathrm{~nm}$. The radius of the conflict area is $100 \mathrm{~nm}$.

Fig. 2, we use the following two simulations to illustrate the similarity between the two models.

The first simulation is run under the same conditions as described in the simulation of Fig. 6. The aircraft in each stream are initially separated by a distance chosen from a uniform distribution on the interval $[5,15] \mathrm{nm}$. Fig. 8 gives a snapshot taken during the conflict resolution process, and also shows the distribution of the 500 tested aircraft with respect to their heading changes. There is significant similarity between the two distributions in Fig. 6 and Fig. 8 . (Note that a heading change of 0.01 radian results in approximately a lateral displacement of $1 \mathrm{~nm}$ lateral displacement after the aircraft flies $100 \mathrm{~nm}$ straight.)

The second simulation uses the heading change model to resolve conflicts for a scenario with uniform arrival geometry. The initial separation distance of aircraft is $S=5 \mathrm{~nm}$. Fig. 9 shows the structure of the resulting traffic flow, which is similar to that observed in Fig. 7 (lower picture).

From the examples presented above, we see that the lateral position change model appears to be a good approximation to the heading change model, especially for very small perturbations.

\section{Comparison with Centralized Resolution Strategies}

The position change model makes it possible and fairly easy to compare the solutions provided by decentralized conflict resolution strategies with centralized, optimal solution strategies that may be obtained for a large but finite set of aircraft. The goal of this study is to evaluate the degree of "inefficiency" of the 


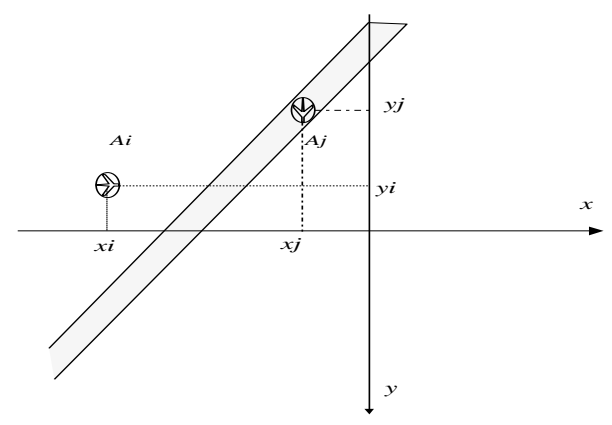

Figure 10: Conflict avoidance constraints.

decentralized conflict resolution scheme discussed in the earlier paragraphs.

Considering two aircraft streams as introduced previously, we assume that the number of aircraft is now finite (the two streams are truncated). The centralized optimization is that of minimizing the maximum lateral deviation experienced by any aircraft, subject to the constraint that all conflicts be solved. Such an optimization problem may be easily written as a mixed integer programming problem, as follows. Referring to Fig. 10, aircraft $A_{i}$ and $A_{j}$ (assumed to travel at the same, constant speed on orthogonal courses) will not be in conflict if and only if the circle $C_{i}$ does not intersect the "shadow" projected by aircraft $A_{j}$. Let $\left(x_{i}, y_{i}\right)$ and $\left(x_{j}, y_{j}\right)$ be the coordinates of aircraft $A_{i}$ and $A_{j}$ and let $d$ be the minimum allowable miss distance. $A_{i}$ and $A_{j}$ are not in conflict if and only if

$$
\begin{array}{r}
x_{i}+y_{i} \geq x_{j}+y_{j}+\sqrt{2} d \\
\text { or } \\
x_{i}+y_{i} \leq x_{j}+y_{j}-\sqrt{2} d .
\end{array}
$$

These constraints are linear in the decision variables $y_{i}$ and $x_{j}$ (which are the lateral deviations for the eastbound and southbound aircraft, respectively). The centralized conflict resolution problem may therefore be written as

$$
\begin{array}{r}
\text { Minimize } \max \left(\max _{A_{i}} \text { is eastbound }\left|y_{i}\right|,\right. \\
\left.\max _{A_{j}} \text { is southbound }\left|x_{j}\right|\right)
\end{array}
$$

Subject to (2),

where the decision variables are $y_{i}$ for the eastbound aircraft and $x_{j}$ for the southbound aircraft $\left(x_{i}\right.$ and $y_{j}$ are fixed parameters). This problem may be easily recast as the mixed-integer linear programming prob-

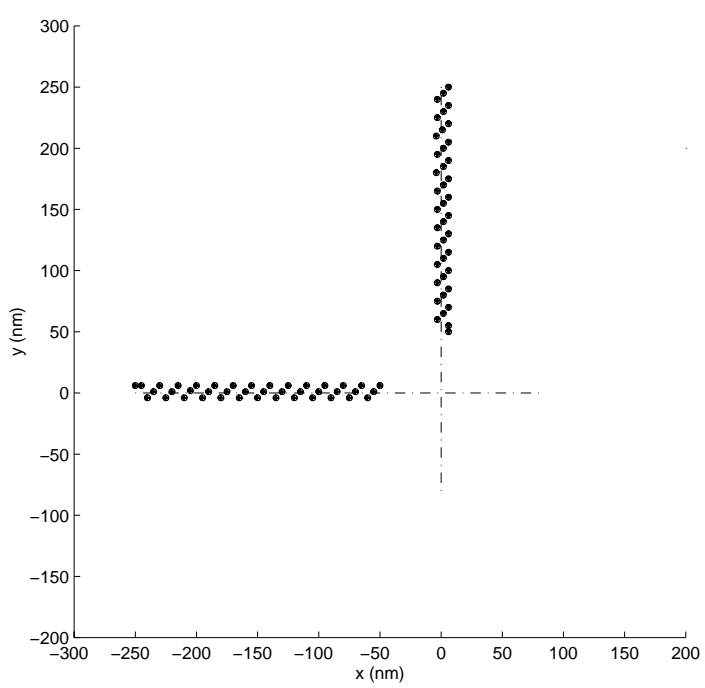

Figure 11: Test case for uniform arrival geometry using the heading change model. The initial separation distance is $5 \mathrm{~nm}$. The radius of the conflict area is $100 \mathrm{~nm}$.

lem

$$
\begin{aligned}
\text { Minimize } & \gamma \\
\text { subject to } & -\gamma \leq y_{i} \leq \gamma, \quad A_{i} \text { is eastbound, } \\
& -\gamma \leq x_{j} \leq \gamma, \quad A_{j} \text { is southbound } \\
& M t_{i j}+x_{i}+y_{i} \geq x_{j}+y_{j}+\sqrt{2} d \\
& M\left(t_{i j}-1\right)+x_{i}+y_{i} \leq x_{j}+y_{j}-\sqrt{2} d
\end{aligned}
$$

where the continuous decision variables are $y_{i}$ for the eastbound aircraft and $x_{j}$ for the southbound aircraft, and $t_{i j}$ are binary decision variables. $x_{i}$ and $y_{j}$ are fixed parameters, and $M$ is a large constant. This problem may be solved efficiently using powerful linear programming optimization software such as CPLEX [4].

Fig. 11 shows the conflict resolution for the two streams of aircraft, with a total of 82 aircraft. In this example, the initial separation distance between aircraft is $S=d=5 \mathrm{~nm}$ and the aircraft are initially configured as described in Fig. 7 (lower picture). Compared with Fig. 7, Fig. 11 reveals a slightly more compact conflict resolution structure: The largest lateral displacement experienced by the aircraft in Fig. 11 is $6.1 \mathrm{~nm}$, which is slightly smaller than $7.1 \mathrm{~nm}$ - the largest displacement of aircraft in the decentralized test.

\section{$\underline{\text { Discussion }}$}

The simplicity of the stability analysis stands with no doubt in contrast with the complexity of the observed aircraft behaviors during simulations: While aircraft conflict avoidance behaviors may display a large number of possible behaviors, it is possible to 
determine an upper bound on the lateral deviation by using first principles. The case study performed here reveals that crucial to the proof of flow stability is the fact that vehicles behave "intelligently" as they attempt to minimize the deviation from their intended path. It is worth noting that statistical approaches such as the one presented in [21], for example, because they perform open-loop analyses, would have resulted in a very large number of predicted conflicts. In comparison, the present analysis, because it is performed on the closed-loop system, predicts no conflict will ever occur and the traffic flow will be handled efficiently (deviations remain bounded). On the other hand, the current analysis pays no attention to robustness issues and what happens in case of delinquent aircraft behavior. Such analysis is the object of current research.

\section{$\underline{\text { Conclusion }}$}

In this paper, we have considered the problem of demonstrating stability of two interacting aircraft flows in a Free-Flight environment when these aircraft obey simple conflict resolution strategies. Using elementary arguments, we have shown for a specific aircraft encounter scenario that these interacting flows remain stable and derived a simple upper bound on the maximum deviation experienced by the aircraft.

Our future research will address the problem of conflict resolution with both speed and heading control. We will consider scenarios with more complex intersecting flow geometry, including the one involving multiple aircraft flow streams.

\section{$\underline{\text { Acknowledgements }}$}

This work was supported by NASA Ames Research Center, under Grant NCC2-1044, and by the Office of Naval Research, under Young Investigator Award N-00014-99-1-0668. The authors would like to thank Sommer Gentry from MIT for performing the centralized control simulations.

\section{$\underline{\text { References }}$}

[1] J.-M. Alliot, N. Durand, and G. Granger, "FACES: a free flight autonomous and coordinated embarked solver," FAA-Eurocontrol Air Traffic Management Seminar, Orlando, 1998.
[2] J. Andrews, "A relative motion analysis of horizontal avoidance," Technical Report, MIT, Lincoln Lab, 1978.

[3] J. Bobick, Improved Navigation by Combining VOR/DME Information with Air of Inertial Data, PhD Thesis, Stanford University, 1972.

[4] ILOG CPLEX user's guide, ILOG, 1999.

[5] S. Devasia and G. Meyer, "Automated conflict resolution procedures for air traffic management," Proc. 38th IEEE Conf. Decision and Control, Phoenix, Arizona, pp. 2456-2462, 1999.

[6] E. Dolginova and N. Lynch, "Safety verification for automated platoon maneuvers: a case study," International Workshop on Hybrid and RealTime Systems (HART'97, Grenoble, France, March 26-28, 1997), vol. 1201, Lecture Notes in Computer Science, Springer-Verlag, pp. 154-170, 1997.

[7] M. S. Eby, "A self-organizational approach for resolving air traffic conflicts," The Lincoln Lab. Journal, vol. 7, no. 2, pp. 239-253, 1994.

[8] E. Frazzoli, Z. -H. Mao, J. -H. Oh, and E. Feron, "Resolution of conflicts involving many aircraft via semidefinite programming," Technical Report ICAT 4-1999, International Center for Air Transportation, MIT, Cambridge, 1999.

[9] R. J. Hansman, "The national airspace system (NAS), how well do we know the plant?" SAE Control Systems Meeting, 1998.

[10] J. Kosecka, C. Tomlin, G. Pappas, and S. Sastry, "Generation of conflict resolution maneuvers for air traffic management," IEEE Int. Conf. Intelligent Robots and Systems, Piscataway, NJ, vol. 3, pp. 1598-1603, 1997.

[11] J. Krozel, T. Mueller, and G. Hunter, "Free flight conflict detection and resolution analysis," AIAA Conf. Guidance, Navigation, and Control, San Diego, CA, 1996.

[12] J. Krozel and M. Peters, "Strategic conflict detection and resolution for free flight," Proc. 36th IEEE Conf. Decision and Control, San Diego, pp. 1822-1828, Dec. 1997.

[13] P. K. Menon, G. D. Sweriduk, and B. Sridhar, "Optimal strategies for free- flight air traffic conflict resolution," Journal of Guidance, Control, and Dynamics, vol. 22, no. 2, pp. 203-211, 1999. 
[14] A. W. Merz, "Optimal aircraft collision avoidance," Joint Automatic Control Conf., Columbus, OH, pp. 449-454, 1973.

[15] W. P. Niedringhaus, "Stream option manager: automated integration of aircraft separation, merging, stream management, and other air traffic control functions," IEEE Trans. Systems, Man, and Cybernetics, vol. 25, no. 9, pp. 12691280, 1995.

[16] J. -H. Oh and E. Feron, "Primal-dual quadratic programming approach to multiple conflict resolution," Proc. of American Control Conference, Philadelphia, pp. 2802-2806, 1998.

[17] R. A. Paielli and H. Erzberger, "Conflict probability estimation for free flight," Journal of Guidance, Control, and Dynamics, vol. 20, no. 3, pp. 588-596, 1997.

[18] M. Prandini, J. Lygeros, A. Nilim, and S. Sastry, "Randomized algorithms for probabilistic aircraft conflict detection," Proc. 38th IEEE Conf. Decision and Control, Phoenix, Arizona, pp. 2444-2449, 1999.

[19] N. Pujet and E. Feron, "Flight plan optimization in flexible air traffic environments," AIAA Conf. Guidance, Navigation, and Control, San Diego, CA, 1996.

[20] RTCA Task Force 3: Free Flight Implementation, Final Report, RTCA, 1995.

[21] R. W. Simpson, Course Notes for 16.72: Air Traffic Control, MIT, 1993.

[22] C. Tomlin, G. J. Pappas, and S. Sastry, "Conflict resolution for air traffic management: a study in multi-agent hybrid systems," Technical Report, University of California at Berkeley, 1996, also in IEEE Trans. Automatic Control, July 1998.

[23] C. Tomlin, G. J. Pappas, and S. Sastry, "Noncooperative conflict resolution," Proc. 36th IEEE Conf. Decision \& Control, San Diego, CA, pp. 1816-1821, Dec. 1997.

[24] P. Varaiya, "Smart cars on smart roads: problems of control," IEEE Trans. Automatic Control, vol. 38, no. 2, pp. 195-207, 1993. 